\title{
Migration et santé à la périphérie de Ouagadougou \\ Une première analyse exploratoire
}

\author{
CLÉMENTINE ROSSIER ${ }^{1,2}$ \\ ABDRAMANE SOURA ${ }^{2}$ \\ BRUNO LANKOANDE ${ }^{2}$
}

\section{Résumé}

En Afrique subsaharienne, les migrations du milieu rural vers la ville restent un phénomène important, pouvant avoir une influence sur les conditions de santé en ville. Dans l'Observatoire de Population de Ouagadougou, Burkina Faso, 71 \% des adultes de plus de 15 ans ne sont pas nés dans la capitale. Utilisant les données collectées dans cet observatoire en 2010, nous étudions le lien entre le statut migratoire et quelques dimensions de la santé (pratiques d'assainissement, malnutrition et risque de fièvre chez les enfants; surpoids, hypertension, dépression et accidents chez les adultes). L'effet des caractéristiques socio-économiques est contrôlé dans cette analyse. Les enfants de migrants ne sont pas plus malnutris ou fiévreux que les enfants de natifs. Les pratiques d'assainissement (ordures et lieu d'aisance) sont tout aussi bonnes dans les ménages dirigés par des migrants. Les adultes migrants sont moins déprimés que les natifs de la capitale, du moins après 10 ans de résidence. Contrairement aux études menées dans d'autres villes africaines, les migrants ne sont pas moins souvent en surpoids ou moins souvent hypertendus à leur arrivée. Ils sont même plus hypertendus que les natifs après avoir résidé longtemps en ville, peut-être à cause d'un retour sélectif au village des migrants qui ne présentent pas de problèmes cardiovasculaires au moment de la retraite. Enfin, les migrants de longue durée déclarent moins d'accidents ayant donné lieu à un recours aux soins, sans doute parce qu'ils consultent moins en cas de problème de santé.

\section{Mots-clés}

Ville, migration, santé, Ouagadougou Afrique au Sud du Sahara.

\footnotetext{
1. Institut National d'Études Démographiques, Paris.

2. Institut Supérieur des Sciences de la Population, Université de Ouagadougou.
} 


\begin{abstract}
Summary
In Sub-Saharan Africa, rural-to-urban migration remains a significant phenomenon, likely to impact urban health conditions. In the Ouagadougou Demographic Surveillance System (DSS), Burkina Faso, $71 \%$ of adults aged 15 or over were not born in the capital. Using survey data collected in this DSS in 2010, we study the link between migration status and some dimensions of health (sanitation practices, malnutrition and risk of fever in children, overweight, hypertension, depression and accidents in adults). The effect of socio-economic characteristics is controlled in this analysis. Children of migrants are not more malnourished or more feverish than children of natives. Sanitation practices (waste and toilet) are equally good in households headed by migrants. Adult migrants are less depressed than those born in the capital city, at least after 10 years of residence. Unlike studies in other African cities, migrants are not less often overweight or less often hypertensive at their arrival in Ouagadougou. They are even more hypertensive than natives after a long time of residence, perhaps due to a selective return to the village of migrants who do not have cardiovascular problems at the time of retirement. Finally, long-term migrants declare fewer accidents which resulted in health care utilization, probably because they visit health services less in case of health problem.
\end{abstract}

\title{
Keywords
}

Urban, migration, health, Ouagadougou, Subsaharian Africa

\section{Introduction $^{3}$}

En Afrique subsaharienne, la migration est une stratégie d'amélioration des conditions de vie pour les populations les plus pauvres. Les migrations du milieu rural vers la ville, qui restent un phénomène important, peuvent ainsi avoir une influence sur les conditions de santé en ville. À la 61ème assemblée mondiale de la santé (19-24 mai 2008, à Genève), la santé des migrants est apparue comme une préoccupation majeure à prendre en compte dans l'agenda plus large des questions de migration et développement (OMS, 2008). Cette assemblée a insisté sur la nécessité de disposer de données empiriques additionnelles sur la santé des migrants et leur accès aux soins afin d'aider à établir des politiques plus efficaces.

3. Cette recherche a été rendue possible grâce au financement de Wellcome Trust de l'OPO de 2008 à 2012 (numéro de financement WT081993MA). Nous remercions également les personnes suivantes pour leurs apports dans la construction du questionnaire de l'enquête santé : Géraldine Duthé, Hélène Deslisle, Pierre Fournier, Valéry Ridde, Alain Levêque, Slim Haddad, Maria Victoria Zunzunegui. 
S'intéressant à la périphérie de Ouagadougou, la capitale du Burkina Faso, l'objectif de ce travail est d'explorer le rôle du statut migratoire face à quelques problèmes de santé typiques des villes africaines : maladies infectieuses et malnutrition chez les enfants, maladies chroniques et accidents chez les adultes. L'influence de la migration sur la santé apparaît comme une question centrale dans ces villes africaines étant donné que la croissance démographique y est due pour une large part à l'immigration du milieu rural (Konseiga et al., 2009). Ainsi, on estime que la moitié de la croissance de Ouagadougou entre les deux derniers recensements (1996 à 2006) est due à la migration, principalement à la migration du milieu rural (Guengant, 2009). Au cours de cette période, la population ouagalaise est passée de 750000 à 1,5 millions d'habitants, et devrait atteindre 5,8 millions de personnes en 2030 (projections moyennes) (Guengant, 2009). Cette croissance urbaine restera sans doute en partie non planifiée puisque près de $30 \%$ de la population de Ouagadougou habite aujourd'hui dans des quartiers dits «non lotis», dépourvus d'assainissement public, d'adduction à l'eau potable, d'écoles et de services de santé publiques (Boyer, Delaunay, 2009).

Ce travail testera l'effet du statut migratoire sur un ensemble d'indicateurs en rapport avec les problèmes de santé cités plus haut, et cela indépendamment d'autres dimensions des inégalités sociales. Il mobilise des données transversales collectées en 2010 dans l'Observatoire de Population de Ouagadougou (OPO), une plateforme de recherche et d'évaluation d'interventions localisée à la périphérie Nord de la ville. Pour la suite, nous présentons d'abord les données ainsi que l'approche méthodologique utilisée. Nous en viendrons ensuite aux résultats et terminerons par la discussion de ces derniers.

\section{Littérature et hypothèses}

Les recherches sur le lien entre migration et santé se focalisent en général sur trois mécanismes d'action, qui prennent effet à différents moments du processus de migration : avant la migration (selection), juste après la migration (disruption), et dans les années qui suivent la migration (acculturation) (Brockerhoff, 1994). Le premier mécanisme est celui des sélections qui s'opèrent à l'occasion des migrations. Il est bien connu qu'à l'exception des populations soumises à des migrations forcées (Miranda et al., 2009), les migrants sont en général plus instruits, plus nantis et en meilleure santé par rapport à ceux qui restent au lieu d'origine. En comparant de manière transversale la santé des migrants 
vers la ville avec celle des populations restées en milieu rural, il est difficile de savoir si les améliorations constatées dans la plupart des cas peuvent être attribuées à la nouvelle résidence en milieu urbain, ou à un effet de sélection. Une première approche consiste, faute de mieux, à contrôler l'effet des caractéristiques socio-démographiques des migrants (Ssengonzi et al., 2002). Cependant, seules des mesures longitudinales des états de santé avant et après la migration permettent de mettre rigoureusement en évidence l'effet de sélection positive (Nauman et al., 2011).

À l'inverse, lorsque l'on compare la santé des migrants du milieu rural à celles des personnes natives de la ville, on observe le plus souvent un effet de composition négatif : les migrants du milieu rural sont d'ordinaire en moins bonne santé que les personnes nées en milieu urbain parce qu'ils proviennent en moyenne de milieux sociaux plus défavorisés (comparés à la moyenne urbaine), et parce que les migrants s'installent plus souvent dans des quartiers plus insalubres (Brockerhoff, 1990). À Ouagadougou, effectivement, les flux migratoires proviennent essentiellement du milieu rural, nettement plus défavorisé, et s'installent plus souvent dans les quartiers périphériques informels (Rossier et al., 2011). Même si les migrants du milieu rural vers la ville sont plus instruits que les personnes qui restent en milieu rural (Beauchemin, Schoumaker, 2005), ils sont en moyenne moins instruits comparés aux natifs du milieu urbain (Schoumaker et al., 2002). Ainsi, dans les quartiers de l'OPO, $61 \%$ des chefs de famille nés en milieu rural sont sans instruction contre $40 \%$ des chefs de famille nés à Ouagadougou. Par ailleurs, les migrants sont en moyenne plus pauvres que les natifs, même si à leur arrivée en ville, ils sont moins souvent au chômage et plus souvent employés du secteur formel par rapport aux natifs (Zourkaleini, Piché, 2007), la migration étant souvent liée à l'opportunité d'un emploi.

Si l'on peut espérer contrôler cet effet de composition en comparant les migrants et les natifs ayant des caractéristiques socio-économiques semblables, un autre biais de sélection (non mesurable) vient entacher la comparaison entre les migrants et les personnes nées en ville : il s'agit de «l'effet du saumon». Les migrants observés à un moment donné en ville ne constituent en effet qu'une partie des migrants, un certain nombre d'entre eux retournant au village. Les rares études qui ont effectué des mesures de santé après le départ des migrants montrent que ceux qui retournent chez eux sont en moins bonne santé que ceux qui restent en ville (Nauman et al., 2011).

Les migrants, lorsqu'ils arrivent en ville, vivent des premiers temps difficiles : ils perdent souvent leurs repères ainsi que leur réseau d'entrai- 
de, et doivent en reconstruire un autre. En cas de maladie, les migrants récents peuvent, du fait de leur manque de familiarité avec leur nouvel environnement, éprouver plus de difficultés à recourir à une structure de soins et à financer le traitement (Stephenson et al., 2003 ; Subaiya, 2007). Ils peuvent également, pour les mêmes raisons, fréquenter moins assidument les centres de santé maternelle et infantile (Kiros, White, 2004). Brokerhoff (1990) montre toute l'importance de ces effets perturbateurs (disruption effects) : s'intéressant à la mortalité des enfants, il constate une augmentation de celle-ci dans les deux années suivant la migration de leur mère en ville (données de 17 Enquêtes Démographiques et de Santé dans les années 1970 et 1980). Plus récemment, Bocquier et al. (2011) mettent en évidence une mortalité plus élevée chez les enfants de migrantes récentes dans les bidonvilles de Nairobi.

Un dernier mécanisme important dans la relation entre migration et santé est celui de l'acculturation. Les habitudes de vie acquises dans le milieu d'origine, différentes des habitudes urbaines, ne sont abandonnées que graduellement par les migrants. Par exemple, des travaux sur les facteurs de risque de maladies cardiovasculaires à Cotonou et Dakar montrent que les migrants du milieu rural sont plus sveltes et moins hypertendus que les citadins à leur arrivée en ville, mais adoptent progressivement un mode alimentaire plus riche faisant en sorte que les écarts avec les natifs diminuent avec leur durée en ville (Sodjinou et al., 2008; Duboz et al., 2011). Notons que ces effets de l'acculturation sont parfois difficiles à distinguer des effets de perturbation. Ainsi, la moindre utilisation des services de santé par les migrants récents, évoquée plus haut, pourrait aussi traduire une préférence pendant un certain temps pour des modes de recours aux soins traditionnels.

Ce travail, comme nous l'avons déjà signalé, étudiera, dans le cas des quartiers de l'OPO, les différences de santé entre les personnes nées en ville et les migrants, en s'intéressant aux problèmes suivants : les maladies infectieuses et la malnutrition chez les enfants, les maladies non transmissibles (hypertension, surpoids, dépression) et les accidents chez les adultes. Les natifs seront comparés aux migrants en fonction de leur durée de résidence, ce qui permettra de mieux repérer d'éventuels effets de perturbation et d'acculturation.

Nous faisons l'hypothèse, en fonction de la littérature existante, que les enfants de migrants ont des indicateurs de santé moins favorables. Les différences qui demeurent, après contrôle des caractéristiques sociodémographiques, pourront être attribuées par déduction à des phénomènes de perturbation et d'acculturation, surtout si les relations évoluent avec la durée de résidence. 
En ce qui concerne les adultes migrants, l'hypothèse est qu'ils sont favorisés par rapport aux natifs de la ville pour des maladies non transmissibles telles que le surpoids et l'hypertension, en raison d'un mode d'alimentation moins favorable à la santé des adultes en milieu urbain. À l'inverse, nous nous attendons à une moins bonne santé mentale et à une plus grande fréquence des accidents chez les migrants récents, compte tenu du stress et de la désorientation des migrants au moment de leur installation en ville (effets de perturbation). À plus long terme, nous faisons l'hypothèse que la fréquence de la mauvaise santé mentale et des accidents des migrants est la même que pour les natifs de la ville.

\section{Données et méthodes}

\section{Milieu et échantillon}

Les analyses sont basées sur des données collectées dans l'Observatoire de Population de Ouagadougou (OPO) qui est une plateforme d'études et d'interventions mise en place en 2008 dans cinq quartiers situés à la périphérie Nord de la ville (Figure 1, Rossier et al., 2012). Parmi ces quartiers, deux sont viabilisés (Kilwin, Tanghin) et trois sont constitués d'habitat informel (Nonghin, Polesgo, Nioko 2).

En début 2010, la population de la zone d'étude était estimée à environ 80000 résidents $^{4}$, soit 14000 ménages. Entre février et juin de la même année, une enquête quantitative transversale a été menée auprès de 1700 ménages afin d'explorer les problèmes de santé spécifiques aux populations urbaines les plus vulnérables, de préparer des évaluations d'interventions et de mener des études plus approfondies sur ces problèmes de santé. La stratégie d'échantillonnage utilisée a conduit à une surreprésentation des ménages contenant des personnes de 50 ans et plus, ce dont nous tenons compte dans l'interprétation de certains résultats.

La population d'étude était constituée des enfants de moins de 5 ans et des adultes de plus de 15 ans. Les enfants de 5 à 14 ans ne sont pas pris en compte par l'enquête. Toutes les personnes éligibles ont été enquêtées dans les ménages échantillonnés. Ainsi, les individus de 15 ans ou plus (au nombre de 2357 ) ont été interrogés sur l'état de santé (tension

4. Les individus résidents sont ceux présents dans la zone d'étude depuis plus de 6 mois. 
artérielle, présence de maladie chronique, limitations fonctionnelles, santé subjective), la pratique d'activité physique, la nutrition, les accidents et violences, l'accès aux soins pour la dernière maladie intervenue dans les 30 derniers jours. Des mesures anthropométriques (poids, taille, tour de taille) ont également été effectuées pour ces adultes. Pour ce qui est des femmes en particulier, un questionnaire complémentaire sur l'utilisation de la contraception et la ménopause leur a été administré. Par ailleurs, les questions sur les limitations fonctionnelles ont été plus détaillées pour les personnes de 50 ans et plus afin d'analyser les facultés cognitives et le soutien économique et social. Pour les enfants de moins de 5 ans (au nombre de 950), les mères ont été interrogées sur le recours aux soins pour le dernier épisode de maladie intervenu dans les deux dernières semaines. Des questions ont également été posées sur l'utilisation de la moustiquaire pour la prévention du paludisme chez l'enfant ainsi que sur les accidents et les actes de violence dont l'enfant a été victime au cours des 12 derniers mois. Comme chez les adultes, leur état nutritionnel a été mesuré au moyen de mesures anthropométriques (poids, taille, périmètre brachial).

FIGURE 1 Localisation des zones suivies par l'Observatoire de Population de Ouagadougou

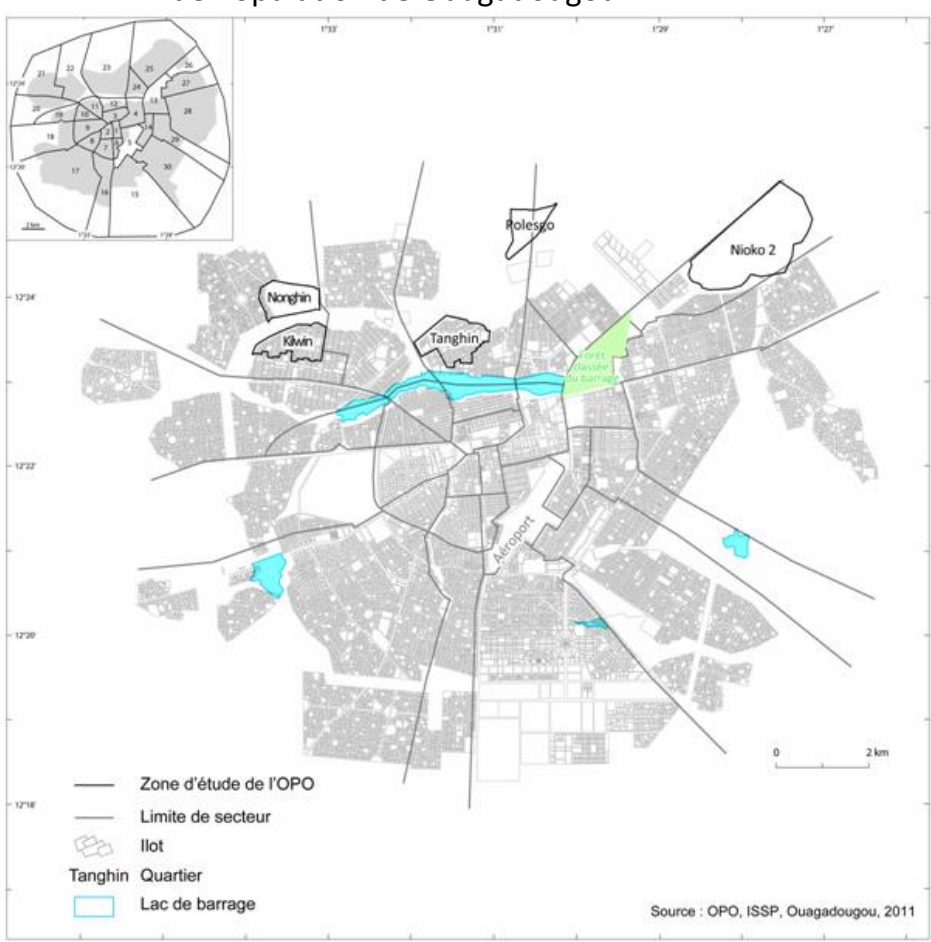




\section{Les variables dépendantes}

Le risque de morbidité infectieuse et de la malnutrition chez l'enfant est analysé ici à travers quatre variables : la survenue de la fièvre au cours des deux dernières semaines chez les enfants de moins de 5 ans, leur risque d'insuffisance pondérale, le type de toilette et le mode d'évacuation des ordures ménagères. Ces deux dernières variables sont mesurées dans tous les ménages de la zone d'étude et servent de proxy pour d'autres morbidités infantiles infectieuses (les maladies diarrhéiques par exemple). Le fait de s'intéresser aux enfants se justifie par leur plus grande exposition aux risques environnementaux, lesquels constituent la principale source de maladies infectieuses.

L'insuffisance pondérale, mesurée par le rapport du poids sur l'âge résume aussi bien les conditions nutritionnelles de court que de long terme dans la mesure où une faible valeur de cet indice peut être provoquée par une perte de poids ou un retard de croissance (Briend, 1998 ; Mariko, 2004). L'indice standardisé de ce rapport poids-pour-âge couramment appelée z-score (Dibley et al., 1987) est utilisé pour détecter les enfants malnutris. Ils s'agit des enfants dont le z-score a une valeur inférieure à - 2 (Dibley et al., 1987). Dans le cas d'une valeur inférieure à - 3, la malnutrition est dite sévère, mais ce dernier cas ne sera pas abordé dans ce travail.

Pour ce qui est du type de toilette, les pratiques habituelles dans les quartiers suivis nous conduisent à retenir deux modalités, à savoir la nature et les autres modes composés à $94 \%$ de latrines. Tenant compte également des pratiques courantes à Ouagadougou, l'évacuation des ordures a été codée en deux modalités : collecte organisée (centre de précollecte ou service de ramassage) ou non. La collecte non organisée est dominée par les dépôts sur les terrains vagues ou au coin de la rue (92\%). En raison de ces dichotomies, nos analyses privilégieront la probabilité d'utiliser la nature comme lieu d'aisance et celle de la collecte des ordures organisée. Nous partons de l'hypothèse que les ménages utilisant la nature comme lieu de toilette ainsi que ceux évacuant les ordures sur des tas d'immondices sont moins hygiéniques et que le risque de maladies infectieuses est plus élevé dans ces ménages.

La charge des maladies non transmissibles, analysée chez les adultes, est approchée par le surpoids, l'hypertension artérielle et la dépression. Le surpoids est mesuré par l'Indice de Masse Corporelle (IMC). Un indice de masse corporelle supérieure à 25 correspond à une situation de surpoids. Seuls les adultes de 20 ans et plus ont été retenus pour les analyses portant sur le surpoids. Les tensions diastoliques et systoliques ont 
été mesurées trois fois chez les répondants de 15 ans et plus à la fin de l'entretien. La moyenne de ces trois mesures a été retenue comme valeur de la tension. Un individu hypertendu est une personne ayant une tension systolique de 140 millimètres de mercure ( $\mathrm{mm} \mathrm{Hg}$ ) ou plus, ou une tension diastolique de 90 millimètres de mercure ou plus. La dépression quant à elle a été mesurée chez les personnes de 15 ans et plus en utilisant le MINI (Mini International Neuropsychiatric Interview) avec deux questions filtres (tristesse/désintérêt) 5 (Sheehan et al., 1998). Une personne déprimée est une personne qui a répondu par l'affirmative à au moins une de ces deux questions, ce qui était suivi de questions supplémentaires pour mieux comprendre les conditions de la dépression. Ces questions ont été traduites en langue locale (le Moré) et n'ont été administrées qu'à des répondants de langue française ou parlant le Moré6.

Enfin, pour ce qui concerne les accidents, ils sont mesurés chez les adultes de 15 ans et plus par la survenue d'un accident ayant nécessité la consultation d'un agent de santé ou d'un service de santé au cours des 12 derniers mois. L'analyse des inégalités devra être ici prudente dans la mesure où ces dernières pourraient également être induites par des différences de recours aux soins.

\section{Les variables indépendantes}

Nous cherchons à décrire la variation dans quelques dimensions de la santé en fonction du statut migratoire des individus enquêtés. Celui-ci est approché par la durée de résidence à Ouagadougou, codée en trois catégories: les natifs de Ouagadougou, les migrants résidents depuis moins de 10 ans et les migrants résidents depuis plus de 10 ans. Des problèmes de petits effectifs n'ont pas permis de distinguer les migrants arrivés plus récemment, c'est-à-dire ceux qui résident à Ouagadougou depuis moins de 5 ans. Dans l'analyse de la fièvre et de la malnutrition chez l'enfant, la variable du statut migratoire se réfère à la mère ${ }^{7}$ tandis

5. Ces deux questions sont : Vous êtes-vous senti(e) déprimé(e), par exemple, triste ou vide, pratiquement toute la journée, et ce, presque tous les jours au cours des deux dernières semaines? Durant ces deux dernières semaines, aviez-vous presque tout le temps le sentiment d'avoir perdu l'intérêt ou le plaisir pour les choses qui vous plaisent habituellement?

6. Seul $0,36 \%$ des adultes de l'échantillon n'ont pas répondu aux questions du module sur la dépression à cause de la contrainte linguistique.

7. La prise en compte des caractéristiques de la mère est une procédure classique dans l'analyse des inégalités de santé de l'enfant. En ce qui concerne les enfants de l'en- 
que dans celle des pratiques d'assainissement (type de toilette et gestion des ordures), elle se réfère au chef de ménage (Tableau 1). Pour les indicateurs de santé des adultes, le statut migratoire est celui de l'individu.

TABleau 1 Variables utilisées dans l'analyse

\begin{tabular}{|c|c|c|c|}
\hline $\begin{array}{l}\text { Variable } \\
\text { dépendante }\end{array}$ & $\begin{array}{l}\text { Niveau } \\
\text { de mesure } \\
\text { et source }\end{array}$ & $\begin{array}{l}\text { Principales } \\
\text { variable } \\
\text { indépendantes }\end{array}$ & Variables de contrôle \\
\hline Fièvre & $\begin{array}{l}\text { Enfants de - de } \\
5 \text { ans (échantil- } \\
\text { lon de l'enquête } \\
\text { santé) }\end{array}$ & $\begin{array}{l}\text { Durée de rési- } \\
\text { dence de la mè- } \\
\text { re à Ouagadou- } \\
\text { gou }\end{array}$ & $\begin{array}{l}\text { Âge et sexe de l'enfant, religion et ethnie } \\
\text { de la mère, niveau de vie du ménage, ni- } \\
\text { veau d'instruction de la mère, type de } \\
\text { quartier (loti/non loti) }\end{array}$ \\
\hline Malnutrition & $\begin{array}{l}\text { Enfants de - de } \\
5 \text { ans (échantil- } \\
\text { lon de l'enquête } \\
\text { santé) }\end{array}$ & $\begin{array}{l}\text { Durée de rési- } \\
\text { dence de la mè- } \\
\text { re à Ouagadou- } \\
\text { gou }\end{array}$ & $\begin{array}{l}\text { Âge et sexe de l'enfant, religion et ethnie } \\
\text { de la mère, niveau de vie du ménage, ni- } \\
\text { veau d'instruction de la mère, type quar- } \\
\text { tier (loti/non loti) }\end{array}$ \\
\hline $\begin{array}{l}\text { Utilisation de } \\
\text { la nature } \\
\text { comme lieu } \\
\text { d'aisance }\end{array}$ & $\begin{array}{l}\text { Ménage (ex- } \\
\text { haustif) }\end{array}$ & $\begin{array}{l}\text { Durée de rési- } \\
\text { dence du chef } \\
\text { de ménage à } \\
\text { Ouagadougou }\end{array}$ & $\begin{array}{l}\text { Âge, sexe, ethnie, religion et niveau d'ins- } \\
\text { truction du chef de ménage, niveau de vie } \\
\text { du ménage, type de quartier (loti/non loti) }\end{array}$ \\
\hline $\begin{array}{l}\text { Collecte des } \\
\text { ordures orga- } \\
\text { nisée }\end{array}$ & $\begin{array}{l}\text { Ménage } \\
\text { (exhaustif) }\end{array}$ & $\begin{array}{l}\text { Durée de rési- } \\
\text { dence du chef } \\
\text { de ménage à } \\
\text { Ouagadougou }\end{array}$ & $\begin{array}{l}\text { Âge, sexe, ethnie, religion et niveau d'ins- } \\
\text { truction du chef de ménage, niveau de vie } \\
\text { du ménage, type de quartier (loti/non loti) }\end{array}$ \\
\hline $\begin{array}{l}\text { Hypertension } \\
\text { artérielle }\end{array}$ & $\begin{array}{l}\text { Adultes de } \\
15 \text { ans et }+ \\
\text { (échantillon de } \\
\text { l'enquête santé) }\end{array}$ & $\begin{array}{l}\text { Durée de rési- } \\
\text { dence de l'indivi- } \\
\text { du à Ouagadou- } \\
\text { gou }\end{array}$ & $\begin{array}{l}\text { Âge, sexe, ethnie, religion, niveau d'ins- } \\
\text { truction de l'individu, niveau de vie du mé- } \\
\text { nage, type de quartier (loti/non loti) }\end{array}$ \\
\hline Surpoids & $\begin{array}{l}\text { Adultes de } \\
20 \text { ans et + } \\
\text { (échantillon de } \\
\text { l'enquête santé) }\end{array}$ & $\begin{array}{l}\text { Durée de rési- } \\
\text { dence de l'indivi- } \\
\text { du à Ouagadou- } \\
\text { gou }\end{array}$ & $\begin{array}{l}\text { Âge, sexe, ethnie, religion, niveau d'ins- } \\
\text { truction de l'individu, niveau de vie du mé- } \\
\text { nage, type de quartier (loti/non loti) }\end{array}$ \\
\hline Dépression & $\begin{array}{l}\text { Adultes de } \\
15 \text { ans et + } \\
\text { (échantillon de } \\
\text { l'enquête santé) }\end{array}$ & $\begin{array}{l}\text { Durée de rési- } \\
\text { dence de l'indivi- } \\
\text { du à Ouagadou- } \\
\text { gou }\end{array}$ & $\begin{array}{l}\text { Âge, sexe, ethnie, religion, niveau d'ins- } \\
\text { truction de l'individu, niveau de vie du mé- } \\
\text { nage, type de quartier (loti/non loti) }\end{array}$ \\
\hline $\begin{array}{l}\text { Accident } \\
\text { ayant nécessi- } \\
\text { té un recours } \\
\text { aux soins }\end{array}$ & $\begin{array}{l}\text { Adultes de } \\
15 \text { ans et + } \\
\text { (échantillon de } \\
\text { l'enquête santé) }\end{array}$ & $\begin{array}{l}\text { Durée de rési- } \\
\text { dence de l'indivi- } \\
\text { du à Ouagadou- } \\
\text { gou }\end{array}$ & $\begin{array}{l}\text { Âge, sexe, ethnie, religion, niveau d'ins- } \\
\text { truction de l'individu, niveau de vie du mé- } \\
\text { nage, type de quartier (loti/non loti) }\end{array}$ \\
\hline
\end{tabular}

quête santé, 92,2 \% ont une mère qui réside dans le ménage et dont nous connaissons les caractéristiques. Environ $16 \%$ des enfants ne résident pas avec leur père; dans ce cas, les caractéristiques des pères sont inconnues dans notre système de suivi démographique. 
Les autres variables indépendantes utilisées pour contrôler l'effet du statut migratoire sont le sexe, le niveau de vie du ménage, le type de quartier (loti/non loti), la religion, l'ethnie et le niveau d'instruction (Tableau 1). Pour ce qui est du niveau de vie, comme cela se fait classiquement dans les études démographiques, un proxy a été construit à partir de la possession de quelques biens d'équipement (téléviseur, réfrigérateur) et du principal moyen de déplacement considéré comme le moyen de déplacement de plus haute valeur possédé par le ménage. L'analyse en composantes principales (ACP) a été utilisée pour déterminer le coefficient attaché à chaque bien. Cet indicateur courant (Filmer, Pritchett, 1999, 2001 ; Lachaud, 2001) n'est pas cependant exempt de critique. Les caractéristiques de l'habitat et les biens d'équipement ne sont pas, par exemple, toujours bien corrélés au niveau de vie mesuré par les dépenses de consommation du ménage (Montgomery et al., 2000). Toutefois, les enquêtes sociodémographiques ont des tailles d'échantillon relativement élevées qui améliorent la puissance des tests basés sur ces indicateurs composites de niveau de vie.

Sur la base des scores du premier facteur de l'ACP (qui explique 50,4 \% de la variance), les ménages ont été classés en trois catégories allant du niveau le plus bas au niveau le plus élevé. Les ménages à niveau de vie bas ne possèdent ni télévision, ni réfrigérateur, ni moto, ni voiture. Ils se déplacent à vélo ou à pied. Leur score factoriel est estimé à - 0,67 (Tableau 2). Ces ménages seront qualifiés de «pauvres» dans la suite du travail. Dans la classe moyenne, la moitié des ménages ont accès à la télévision, et près de 9 ménages sur 10 utilisent la mobylette comme principal moyen de déplacement. Le réfrigérateur et la voiture sont cependant rares dans ce groupe. En ce qui concerne la classe sociale la plus favorisée, elle est caractérisée par une proportion très élevée de téléviseurs $(99,7 \%)$ et de réfrigérateurs $(83,6 \%)$. La moto et la voiture sont représentées dans ce groupe à hauteur de $50 \%$ chacune.

TAbleau 2 Caractérisation des classes de niveau de vie

\begin{tabular}{|l|c|c|c|}
\hline $\begin{array}{l}\text { Caractéristiques de } \\
\text { l'habitat et biens d'équi- }\end{array}$ & \multicolumn{3}{|c|}{ Niveau de vie } \\
\hline $\begin{array}{l}\text { pement du ménage } \\
\text { Réfrigérateur }\end{array}$ & Score : - 0,67 & Score : $0,13-1,84$ & Score : $1,92-2,64$ \\
\hline Téléviseur & $0 \%$ & $1,81 \%$ & $83,59 \%$ \\
\hline Moyen de déplacement & $0 \%$ & $49,80 \%$ & $99,73 \%$ \\
\hline À pied ou vélo & $100 \%$ & $11,65 \%$ & $0 \%$ \\
\hline Moto & $0 \%$ & $87,71 \%$ & $49,86 \%$ \\
\hline Voiture & $0 \%$ & $0,63 \%$ & $50,14 \%$ \\
\hline Effectif $(n)$ & 7270 & 6159 & 112 \\
\hline
\end{tabular}


Parmi les autres variables de contrôle, le niveau d'instruction a été codé en trois catégories (aucun, primaire, secondaire et plus). En raison des petits effectifs (et de la grande prédominance de l'ethnie Mossi dans les quartiers suivis), il n'est pas possible de distinguer les différents groupes ethniques du pays; deux catégories seulement ont donc été retenues à savoir les Mossi et les autres ethnies. De même, pour ce qui est de la religion, le christianisme a été distingué des autres confessions du fait que, comparés aux musulmans et aux animistes, les chrétiens ont en général un comportement de santé meilleur en Afrique subsaharienne (Akoto, 1990). Enfin, lorsque la variable âge se rapporte à l'enfant, elle est calculée par année (sans regroupement en classes) tandis que lorsqu'elle se rapporte à l'adulte, elle a été regroupée en 4 classes (moins de 35 ans, 35-44 ans, 45-54 ans et 55 ans et plus).

\section{Méthodes d'analyse}

Deux méthodes statistiques classiques ont été essentiellement utilisées pour tester les relations : le test du khi-deux pour les relations brutes et la régression logistique pour les relations nettes. Le test du khi-deux, couramment utilisé, est un test d'indépendance entre deux variables nominales qui fournit une présomption sur le niveau d'influence de chaque variable explicative sur la variable dépendante. Quant à la régression logistique, elle consiste à modéliser le logarithme du rapport de cotes (rapport de chance) sous la forme d'une fonction linéaire des variables explicatives. Pour ce qui est des interprétations, l'exponentielle de chaque coefficient indique, pour les caractéristiques nominales, le rapport de cotes entre la modalité correspondante et la modalité de référence, toutes choses égales par ailleurs. Dans le cas des caractéristiques continues, l'exponentielle du coefficient indique le rapport de cotes lorsque la variable concernée augmente d'une unité de valeur, toutes choses égales par ailleurs. 


\section{Résultats}

Les relations bivariées

Risque de morbidité infectieuse et malnutrition

Les premières estimations indiquent que $43,1 \%$ des enfants ont souffert de fièvre au cours des deux semaines précédant l'enquête. La prévalence de l'insuffisance pondérale est estimée à 20,5\% chez les enfants de moins de 5 ans. En ce qui concerne les pratiques d'assainissement, considérées ici comme des facteurs de morbidité infectieuses, 6,7 \% des ménages utilisent la nature comme lieu d'aisance et 19,1\% des ménages pratiquent la collecte des ordures organisée.

L'analyse bivariée de la fièvre et de la malnutrition chez l'enfant ne fait pas ressortir d'inégalités basées sur le statut migratoire de la mère (Tableau 3). Les enfants de natives semblent souffrir un peu moins de fièvre $(40,5 \%)$ comparées aux enfants de migrantes de moins de 10 ans $(43,2 \%)$ ou de plus de 10 ans $(44,7 \%)$, mais ces différences ne sont pas significatives. De même, les enfants de mères natives de Ouagadougou semblent souffrir un peu moins d'insuffisance pondérale $(18,2 \%)$ comparativement à ceux de mères migrantes $(19,7 \%$ quand elles sont arrivées il y a moins de 10 ans et $21,5 \%$ quand elles sont arrivées il y a plus de 10 ans), mais les fluctuations d'échantillons font que ces différences ne sont pas significatives (Tableau 3).

Les pratiques d'assainissement montrent quant à elles que les ménages de migrants utilisent moins la nature comme lieu d'aisance $(5,9 \%$ pour les migrants récents, 6,6\% pour ceux de longue durée contre 7,6\% pour les natifs de Ouagadougou, Tableau 4). L'avantage des migrants récents est encore curieusement observé avec le mode d'évacuation des ordures : ils utilisent plus le service de ramassage ou les centres de précollecte $(24 \%)$ comparativement aux autres $(21,4 \%$ pour les ménages dirigés par des natifs et 18,2\% pour ceux dirigés par des migrants de longue durée). Ce résultat pourrait découler d'éventuels effets de confusion, ce qui sera vérifié dans l'analyse multivariée. 
TABLEAU 3 Relations bivariées entre variables explicatives et occurrence de la fièvre et de l'insuffisance pondérale chez les enfants

\begin{tabular}{|c|c|c|}
\hline Variables & $\begin{array}{l}\text { \% d'enfants ayant eu } \\
\text { la fièvre au cours des } \\
2 \text { dernières semaines }\end{array}$ & $\begin{array}{l}\% \text { d'enfants souffrant } \\
\text { d'insuffisance pondérale }\end{array}$ \\
\hline \multicolumn{3}{|l|}{ Durée de résidence de la mère } \\
\hline Native de Ougadougou & 40,5 & 18,2 \\
\hline Moins de 10 ans & 43,2 & 19,7 \\
\hline 10 ans et + & 44,7 & 21,5 \\
\hline \multicolumn{3}{|l|}{ Sexe de l'enfant } \\
\hline Masculin & 41,5 & 21,2 \\
\hline Féminin & 44,6 & 19,7 \\
\hline \multicolumn{3}{|l|}{ Niveau d'éducation de la mère } \\
\hline Aucun & 43,8 & 20,9 \\
\hline Primaire & 43,6 & 20,4 \\
\hline Secondaire et + & 39,9 & 19,0 \\
\hline \multicolumn{3}{|l|}{ Ethnie de la mère } \\
\hline Mossi & 42,9 & 20,7 \\
\hline Autre & 45,2 & 17,8 \\
\hline \multicolumn{3}{|l|}{ Religion de la mère } \\
\hline Chrétienne & 44,3 & 17,7 \\
\hline Autre & 42,5 & 22,0 \\
\hline \multicolumn{3}{|l|}{ Niveau de vie du ménage } \\
\hline Bas & 43,8 & 21,4 \\
\hline Moyen & 42,8 & 21,4 \\
\hline Élevé & 39,6 & 4,6 \\
\hline \multicolumn{3}{|l|}{ Type de quartier } \\
\hline Loti & 37,6 & 15,2 \\
\hline Non loti & 46,4 & 23,7 \\
\hline Toutes catégories confondues & 43,1 & 20,5 \\
\hline
\end{tabular}

NB : Aucune différence significative.

Parmi les facteurs de contrôle, le niveau d'instruction est lié positivement à de meilleures pratiques d'assainissement (Tableau 4). Le niveau de vie montre également, conformément à nos attentes, une relation positive avec les pratiques d'assainissement et négative avec l'insuffisance pondérale chez les enfants (Tableaux 3 et 4). La résidence dans les quartiers non lotis est associée à une utilisation plus fréquente de la nature comme lieu de toilette, et à un risque plus élevé de fièvre et de malnutrition chez l'enfant. En revanche, la pratique de la collecte organisée des ordures reste très rare dans ce type de quartier. Elle est par contre plus fréquente dans les ménages dirigés par des femmes. Ces derniers utilisent plus la nature comme lieu de toilette. Les ménages dirigés par des chefs plus jeunes ( $<35$ ans) utilisent également plus la nature comme 
lieu de toilette. Ils pratiquent moins la collecte organisée des ordures. Dans les familles dirigées par un Mossi, on utilise plus la nature comme lieu d'aisance et moins le service de ramassage des ordures ou les centres de précollecte. Enfin, en lien avec la religion, les résultats montrent que les chrétiens ont une gestion des ordures plus salubre.

TABLEAU 4 Relations bivariées entre variables explicatives et pratiques d'assainissement des ménages

\begin{tabular}{|c|c|c|}
\hline Variables & $\begin{array}{c}\% \text { de ménages utilisant } \\
\text { la nature comme lieu } \\
\text { d'aisance }\end{array}$ & $\begin{array}{c}\text { \% de ménages prati- } \\
\text { quant la collecte } \\
\text { organisée des ordures }\end{array}$ \\
\hline Durée de résidence du chef de ménage & $*$ & $* * *$ \\
\hline Natif de Ougadougou & 7,6 & 21,4 \\
\hline Moins de 10 ans & 5,9 & 24,0 \\
\hline 10 ans et + & 6,6 & 18,2 \\
\hline Sexe du chef de ménage & $* * *$ & $* *$ \\
\hline Masculin & 6,2 & 18,8 \\
\hline Féminin & 10,5 & 21,2 \\
\hline Groupe d'âge du chef de ménage & $* * *$ & $* * *$ \\
\hline$<35$ ans & 8,2 & 13,1 \\
\hline $35-44$ ans & 5,1 & 21,5 \\
\hline $45-54$ ans & 5,4 & 27,6 \\
\hline 55 ans et + & 6,7 & 24,6 \\
\hline Niveau d'éducation du chef de ménage & $* * *$ & $* * *$ \\
\hline Aucun & 8,2 & 11,7 \\
\hline Primaire & 6,6 & 18,8 \\
\hline Secondaire et + & 3,6 & 36,3 \\
\hline Ethnie du chef de ménage & $* * *$ & $* * *$ \\
\hline Mossi & 7,1 & 17,5 \\
\hline Autre & 3,4 & 34,8 \\
\hline Religion du chef de ménage & $* *$ & $* * *$ \\
\hline Chrétienne & 7,3 & 21,2 \\
\hline Autre & 6,4 & 17,8 \\
\hline Niveau de vie du ménage & $* * *$ & $* * *$ \\
\hline Bas & 11,4 & 5,9 \\
\hline Moyen & 2,4 & 24,9 \\
\hline Élevé & 0,1 & 73,1 \\
\hline Type de quartier & $* * *$ & $* * *$ \\
\hline Loti & 0,9 & 46,7 \\
\hline Non loti & 10,5 & 1,2 \\
\hline Toutes catégories confondues & 6,7 & 19,1 \\
\hline
\end{tabular}

NB : * $\mathrm{p}<0,1 ; * * \mathrm{p}<0,05 ; * * * \mathrm{p}<0,001$. 
TABLEAU 5 Relations bivariées entre variables explicatives et indicateurs de santé non transmissible chez les adultes

\begin{tabular}{|c|c|c|c|c|}
\hline Variables & $\begin{array}{l}\% \text { de per- } \\
\text { sonnes hy- } \\
\text { pertendues } \\
\text { (15 ans et }+ \text { ) }\end{array}$ & $\begin{array}{l}\% \text { de per- } \\
\text { sonnes en } \\
\text { surpoids } \\
(20 \text { ans et }+)\end{array}$ & $\begin{array}{c}\% \text { de per- } \\
\text { sonnes } \\
\text { déprimées } \\
\text { (15 ans et +) }\end{array}$ & $\begin{array}{l}\% \text { de person- } \\
\text { nes ayant eu } \\
\text { un accident } \\
\text { qui a nécessi- } \\
\text { té un recours } \\
\text { aux soins } \\
\text { (15 ans et }+ \text { ) }\end{array}$ \\
\hline Durée de résidence de l’individu & $* * *$ & & & $* *$ \\
\hline Natif de Ouagadougou & 8,1 & 26,7 & 5,9 & 11,5 \\
\hline Moins de 10 ans & 5,3 & 28,3 & 5,3 & 8,8 \\
\hline 10 ans et + & 19,4 & 25,6 & 3,9 & 6,8 \\
\hline Sexe de l'individu & & $* * *$ & & $* * *$ \\
\hline Masculin & 14,5 & 17,5 & 3,9 & 11,0 \\
\hline Féminin & 15,6 & 33,6 & 5,3 & 6,0 \\
\hline Groupe d'âges de l'individu & $* * *$ & $* * *$ & & $* *$ \\
\hline$<35$ ans & 3,8 & 23,7 & 4,1 & 9,7 \\
\hline $35-44$ ans & 18,1 & 34,0 & 4,9 & 8,5 \\
\hline $45-54$ ans & 26,7 & 35,8 & 5,3 & 6,6 \\
\hline 55 ans et + & 44,4 & 18,0 & 5,9 & 4,1 \\
\hline Niveau d'éducation de l'individu & $* * *$ & $* *$ & & $* * *$ \\
\hline Aucun & 21,0 & 23,9 & 5,3 & 5,7 \\
\hline Primaire & 10,9 & 26,4 & 4,2 & 10,5 \\
\hline Secondaire et + & 6,7 & 31,4 & 3,5 & 11,7 \\
\hline Ethnie de l'individu & & & $* *$ & \\
\hline Mossi & 15,3 & 28,8 & 4,2 & 8,6 \\
\hline Autre & 12,1 & 28,8 & 8,6 & 5,8 \\
\hline Religion de l'individu & $* *$ & & & $*$ \\
\hline Chrétienne & 13,1 & 26,3 & 3,7 & 9,8 \\
\hline Autre & 16,4 & 25,9 & 5,2 & 7,2 \\
\hline Niveau de vie de l'individu & & $* * *$ & & \\
\hline Bas & 15,8 & 18,0 & 4,9 & 7,5 \\
\hline Moyen & 15,3 & 29,9 & 4,8 & 8,7 \\
\hline Élevé & 11,0 & 43,9 & 2,5 & 9,6 \\
\hline Type de quartier & $*$ & $* * *$ & $*$ & $* *$ \\
\hline Loti & 19,6 & 32,2 & 3,7 & 6,9 \\
\hline Non loti & 13,1 & 20,0 & 5,6 & 9,8 \\
\hline Toutes catégories confondues & 15,0 & 26,0 & 4,6 & 8,3 \\
\hline
\end{tabular}

NB : ${ }^{*} \mathrm{p}<0,1 ;{ }^{* *} \mathrm{p}<0,05 ; * * * \mathrm{p}<0,001$.

Problèmes de santé non transmissibles et accidents

L'analyse des problèmes de santé non transmissibles (Tableau 5) montre que $15 \%$ des adultes enquêtés étaient hypertendus, $26 \%$ en surpoids et $4,6 \%$ déprimés. Au cours des 12 derniers mois, 8,3\% de ces personnes ont été victimes d'un accident qui a donné lieu à une consul- 
tation médicale. La moitié de ces accidents ont eu lieu sur la voie publique, et peuvent être imputés à la circulation.

Le surpoids n'est pas associé au statut migratoire de l'individu. Les migrants de longue durée sont moins souvent déprimés, mais la différence n'est pas significative. L'examen des accidents montre une fréquence plus élevée chez les natifs de Ouagadougou, et celui de l'hypertension révèle moins d'hypertendus parmi les migrants récents $(5,3 \%)$ et les natifs de la capitale $(8,1 \%)$, contre $19 \%$ pour les migrants de plus de 10 ans.

Au-delà des variables du statut migratoire, les autres facteurs montrent également de nombreuses relations avec les indicateurs de santé étudiés (Tableau 5). Il apparait que le niveau d'instruction est associé négativement à l'hypertension. Le surpoids est moins fréquent parmi les jeunes adultes (<35 ans) et parmi les plus âgés ( $>55$ ans). Le risque d'hypertension augmente avec l'âge contrairement au risque d'accident qui diminue avec l'âge, ce qui est un résultat attendu. Les accidents ayant donné lieu à une consultation médicale sont plus fréquents parmi les personnes les plus instruites et dans les quartiers non lotis. La dépression, l'hypertension et le surpoids sont quant à elles plus rares dans ce type de quartier. Chez les adultes, les Mossi semblent déclarer moins de dépression. Par rapport aux hommes, les femmes ont été moins victimes d'accidents ayant entrainé une consultation médicale. À l'inverse, elles sont plus en surpoids que les hommes.

\section{Les relations nettes}

Risque de morbidité infectieuse et malnutrition

Les résultats multivariés confirment que, contrairement à ce que l'on pourrait attendre, les migrants arrivés il y a plus de 10 ans ont des pratiques plus saines que les natifs de Ouagadougou en ce qui concerne le lieu de toilette (Tableau 6). Le rapport de cotes d'utiliser la nature comme lieu d'aisance est en effet estimé à 0,73 lorsque ces migrants sont comparés aux natifs. Ces derniers ne sont pas différents des migrants récents (durée $<10$ ans) dans leur propension d'utiliser la nature comme lieu de toilette. Les résultats en matière d'évacuation des ordures (Tableau 6) ne montrent pas quant à eux de différence significative basée sur le statut migratoire. 
TABLEAU 6 Rapports de chance des variables explicatives en matière de pratiques d'assainissement des ménages

\begin{tabular}{|c|c|c|}
\hline Variables & $\begin{array}{l}\text { Utilisation de la nature } \\
\text { comme lieu d'aisance }\end{array}$ & $\begin{array}{l}\text { Utilisation de la collecte } \\
\text { des ordures organisées }\end{array}$ \\
\hline \multicolumn{3}{|c|}{ Durée de résidence du chef de ménage } \\
\hline Natif de Ougadougou & 1 & 1 \\
\hline Moins de 10 ans & 0,91 & 0,92 \\
\hline 10 ans et + & $0,73 * * *$ & 1,11 \\
\hline \multicolumn{3}{|l|}{ Sexe du chef de ménage } \\
\hline Masculin & 1 & 1 \\
\hline Féminin & $1,47^{* * *}$ & $1,44 * * *$ \\
\hline \multicolumn{3}{|c|}{ Groupe d'âges du chef de ménage } \\
\hline$<35$ ans & 1 & 1 \\
\hline $35-44$ ans & $0,72 * * *$ & 1,06 \\
\hline $45-54$ ans & 0,85 & 1,06 \\
\hline 55 ans et + & 1,02 & 1,02 \\
\hline \multicolumn{3}{|c|}{ Niveau d'éducation du chef de ménage } \\
\hline Aucun & 1 & 1 \\
\hline Primaire & 0,95 & $1,56 * * *$ \\
\hline Secondaire et + & $0,79 * *$ & $2,02 * * *$ \\
\hline \multicolumn{3}{|l|}{ Ethnie du chef de ménage } \\
\hline Mossi & 1 & 1 \\
\hline Autre & $0,71 * *$ & $1,46 * * *$ \\
\hline \multicolumn{3}{|c|}{ Religion du chef de ménage } \\
\hline Chrétienne & 1 & 1 \\
\hline Autre & $0,78 * * *$ & $1,17^{* *}$ \\
\hline \multicolumn{3}{|l|}{ Niveau de vie du ménage } \\
\hline Bas & 1 & 1 \\
\hline Moyen & $0,30 * * *$ & 2,38 \\
\hline Élevé & $0,04 * * *$ & 6,89 \\
\hline \multicolumn{3}{|l|}{ Type de quartier } \\
\hline Loti & 1 & 1 \\
\hline Non loti & $7,21 * * *$ & $0,02 * * *$ \\
\hline
\end{tabular}

Malgré les différences dans les gestes sanitaires des parents (latrines, gestion des ordures) en fonction de leur niveau d'instruction et de leur niveau de vie (Tableaux 4 et 6), l'occurrence de fièvres chez les enfants de moins de cinq ans au cours des deux semaines précédant l'enquête n'est liée à aucune de ces caractéristiques, ni au statut migratoire (Tableau 7). Les seules différences qui apparaissent sont celles d'une plus grande probabilité de fièvre en zone non lotie (rapport de chance $=$ 1,48 ) et d'une probabilité de fièvre diminuant en fonction de l'âge de l'enfant (rapport de chance $=0,75$ ). 
TABLEAU 7 Rapports de chance des variables explicatives en termes de risques de fièvre et d'insuffisance pondérale chez les enfants

\begin{tabular}{|c|c|c|}
\hline Variables & $\begin{array}{c}\text { Fièvre au cours des } 2 \\
\text { dernières semaines }\end{array}$ & Insuffisance pondérale \\
\hline \multicolumn{3}{|c|}{ Durée de résidence de la mère } \\
\hline Native de Ougadougou & 1 & 1 \\
\hline Moins de 10 ans & 0,94 & 1,10 \\
\hline 10 ans et + & 1,17 & 1,10 \\
\hline \multicolumn{3}{|l|}{ Sexe de l'enfant } \\
\hline Masculin & 1 & 1 \\
\hline Féminin & 1,14 & 0,91 \\
\hline \multicolumn{3}{|c|}{ Niveau d'éducation de la mère } \\
\hline Aucun & 1 & 1 \\
\hline Primaire & 0,95 & 0,97 \\
\hline Secondaire et + & 0,86 & 1,07 \\
\hline \multicolumn{3}{|l|}{ Ethnie de la mère } \\
\hline Mossi & 1 & 1 \\
\hline Autre & 0,81 & 0,99 \\
\hline \multicolumn{3}{|l|}{ Religion de la mère } \\
\hline Chrétienne & 1 & 1 \\
\hline Autre & 1,1 & 0,79 \\
\hline \multicolumn{3}{|l|}{ Niveau de vie du ménage } \\
\hline Bas & 1 & 1 \\
\hline Moyen & 1,04 & 1,12 \\
\hline Élevé & 1,24 & $0,24^{*}$ \\
\hline \multicolumn{3}{|l|}{ Type de quartier } \\
\hline Loti & 1 & 1 \\
\hline Non loti & $1,48 * *$ & $1,44^{*}$ \\
\hline Âge de l'enfant & $0,75^{* * *}$ & $0,62 * * *$ \\
\hline
\end{tabular}

La résidence en zone non lotie expose à de nombreuses maladies infectieuses dont la fréquence empêche la prise de poids chez les enfants, comme en témoigne l'association mise en évidence entre leur risque d'insuffisance pondérale et le type de quartier (Tableau 7). La relation avec le niveau de vie va dans le sens attendu montrant que, comparés aux enfants de niveau de vie bas, ceux de niveau de vie élevé ont un risque d'insuffisance pondérale plus faible (rapport de chance $=0,24$ ). Entre les ménages de niveau de vie moyen et ceux de niveau de vie bas, le risque de malnutrition de l'enfant est le même. Le manque de finesse dans les proxys de niveau de vie construits avec les caractéristiques de l'habitat et les biens d'équipement pourrait être à l'origine de cette faiblesse de relation avec le risque de malnutrition. Ce risque est égale- 
ment le même lorsque les natifs sont comparés aux migrants quelle que soit leur durée de résidence à Ouagadougou. Les autres variables de contrôle ne sont pas significativement liées au risque de malnutrition de l'enfant : aucune différence basée sur le sexe de ce dernier, la religion ou l'ethnie de sa mère n'est observée dans le risque d'insuffisance pondérale.

Problèmes de santé non transmissibles et accidents

Les migrants, quelle que soit leur durée, ont la même probabilité que les natifs d'être en surpoids (Tableau 8). Les migrants de longue durée (>10 ans) sont plus souvent hypertendus (rapport de chance $=1,66$ ). Ils font à l'inverse moins souvent état de symptômes dépressifs (rapport de chance $=0,45$ ) et déclarent également moins d'accidents (rapport de chance $=0,69$ ).

En ce qui concerne les autres variables indépendantes, ni le niveau de vie, ni le niveau d'instruction ne sont liés à l'hypertension. Par contre l'âge, comme on s'y attend, est très fortement lié à cette maladie, les 55 ans et plus ayant environ 19 fois plus de risque d'être hypertendus que les moins de 35 ans (Tableau 8). Dans le cas de la dépression, ni le niveau de vie du ménage ni le niveau d'instruction ne semblent non plus jouer de rôle. Par contre, le fait d'habiter en zone non lotie est lié à une propension plus grande à la dépression, ce qui indique peut-être un lien avec des dimensions de la pauvreté mal mesurées par le proxy de niveau de vie du ménage ${ }^{8}$. Les répondants de quartiers non lotis déclarent également plus d'accidents. Ces accidents sont encore plus fréquents chez les personnes les plus instruites, sans doute aussi parce qu'elles ont plus recours aux soins médicaux.

Les relations avec le surpoids (Tableau 8) montrent que les femmes sont plus à risque que les hommes, ce qui constitue un résultat conforme à nos attentes compte tenu du style de vie sédentaire de beaucoup de citadines au foyer. Les autres relations vont également dans le sens atten$\mathrm{du}$ : les individus plus riches sont plus à risque de surpoids que les autres, et les personnes habitant dans les zones non loties sont moins à risque de surpoids, peut-être parce que ces quartiers capturent des dimensions de la pauvreté non mesurée par notre proxy de niveau de vie du ménage ou alors parce ce que les maladies infectieuses plus fréquen-

8. Une analyse plus poussée de la dépression avec les mêmes données montre que la dépression est associée à un indicateur de la pauvreté des ménages incluant plus de biens, et avec l'insécurité alimentaire des ménages (Duthé et al. 2013). 
tes en zone non lotie empêchent les prises de poids également chez les adultes. Un niveau d'instruction élevé n'est pas lié au surpoids.

TABLEAU 8 Rapports de chance des variables explicatives en termes de santé des adultes

\begin{tabular}{|c|c|c|c|c|}
\hline Variables & Hypertension & Surpoids & Dépression & Accident \\
\hline \multicolumn{5}{|c|}{ Durée de résidence de l'individu } \\
\hline Natif de Ouagadougou & 1 & 1 & 1 & 1 \\
\hline Moins de 10 ans & 0,83 & 1,13 & 0,79 & 0,76 \\
\hline 10 ans et + & $1,66 * *$ & 1,06 & $0,45 * * *$ & $0,69 *$ \\
\hline \multicolumn{5}{|l|}{ Sexe du chef de ménage } \\
\hline Masculin & 1 & 1 & 1 & 1 \\
\hline Féminin & 1,26 & $2,52 * * *$ & 1,42 & $0,56 * *$ \\
\hline \multicolumn{5}{|c|}{ Groupe d'âges du chef de ménage } \\
\hline$<35$ ans & 1 & 1 & 1 & 1 \\
\hline $35-44$ ans & $5,29 * * *$ & $1,85^{* * *}$ & 1,31 & 0,95 \\
\hline $45-54$ ans & $8,76 * * *$ & $1,95 * * *$ & 1,64 & 0,83 \\
\hline 55 ans et + & $18,71^{* * *}$ & 0,74 & $1,78^{*}$ & 0,62 \\
\hline \multicolumn{5}{|c|}{ Niveau d'éducation du chef de ménage } \\
\hline Aucun & 1 & 1 & 1 & 1 \\
\hline Primaire & 1,08 & 1,14 & 0,83 & $1,57^{*}$ \\
\hline Secondaire et + & 0,77 & 1,21 & 0,76 & $1,77^{* *}$ \\
\hline \multicolumn{5}{|l|}{ Ethnie du chef de ménage } \\
\hline Mossi & 1 & 1 & 1 & 1 \\
\hline Autre & 0,83 & 0,84 & $2,73 * * *$ & 0,62 \\
\hline \multicolumn{5}{|l|}{ Religion du chef de ménage } \\
\hline Chrétienne & 1 & 1 & 1 & 1 \\
\hline Autre & $1,52 * *$ & 1,14 & $1,51^{*}$ & 0,75 \\
\hline \multicolumn{5}{|l|}{ Niveau de vie du ménage } \\
\hline Bas & 1 & 1 & 1 & 1 \\
\hline Moyen & 1,08 & $1,71 * * *$ & 1,15 & 1,20 \\
\hline Élevé & 0,77 & $2,53 * * *$ & 0,71 & 1,57 \\
\hline \multicolumn{5}{|l|}{ Type de quartier } \\
\hline Loti & 1 & 1 & 1 & 1 \\
\hline Non loti & 0,90 & $0,72^{* *}$ & $1,74^{* *}$ & $1,94^{* *}$ \\
\hline
\end{tabular}

\section{Discussion}

Les enfants de migrants ne sont pas désavantagés du point de vue de la santé à Ouagadougou, toutes choses égales par ailleurs. Leurs risques de fièvre et de malnutrition sont les mêmes que ceux des enfants de natifs. D'autres analyses de l'enquête santé ont montré que les migrants recourent tout autant aux soins pour leurs enfants que les natifs de la ville 
(Nikiema et al., 2011). Également, les chefs de ménages migrants adoptent les mêmes pratiques de gestion des ordures que les chefs de ménages nés dans la capitale, et tendraient même à adopter des pratiques plus sanitaires en ce qui concerne le lieu d'aisance. Ce dernier résultat s'explique sans doute par le fait que le recours à la nature comme toilette est un comportement qui concerne presque exclusivement les quartiers non lotis dans la zone d'étude. Or, dans ces quartiers, les «natifs de Ouagadougou» sont souvent originaires des anciens villages qui ont été entre temps engloutis par la ville grandissante. Ces anciennes concessions villageoises bénéficient apparemment moins souvent de latrines comparées aux nouvelles habitations du non loti.

L'ensemble des résultats vont dans le sens d'une absence de relation entre le statut migratoire et la santé des enfants dans les quartiers de l'OPO. Ces résultats rejoignent ceux d'une étude en Inde, qui montre une absence de relation entre statut migratoire de la mère et mortalité des enfants après contrôle du statut socio-économique et de la fréquentation des services de santé (Stephenson et al., 2003). De même, une étude en Ouganda montre que le statut migratoire n'explique qu'une faible partie de la variance dans la mortalité des enfants (Ssengonzi et al., 2002). Par contre, la mortalité des enfants de migrants est plus élevée dans les bidonvilles de Nairobi (Konseiga et al., 2009).

Comment expliquer que, du point de vue de la santé, les enfants des migrants ne soient pas désavantagés à Ouagadougou? Plusieurs hypothèses s'offrent à nous. Tout d'abord, la faiblesse des effectifs n'a pas permis d'isoler dans ce travail les migrants très récents. Or, une grande partie de l'explication de la mauvaise santé des enfants de migrants (toutes choses égales par ailleurs) est liée à des phénomènes de perturbations (disruption effects) qui agissent au cours des toutes premières années après la migration, et que nous ne capturons peut-être pas ici. Une autre hypothèse est que les mères ou les familles migrantes avec des enfants maladifs ou qui adoptent moins rapidement les habitudes de la ville retournent plus souvent à leur lieu d'origine. Enfin, une analyse qualitative de la pauvreté dans les quartiers de l'OPO a montré que les migrants ont des réseaux de relations et d'entraide qui semblent aussi développés que les natifs. Les familles migrantes semblent être en contact dès leur arrivée avec des familles natives, ce qui leur permet peutêtre d'acquérir rapidement les savoirs leur permettant de promouvoir la santé de leurs enfants (acculturation rapide) et d'atténuer les effets perturbateurs liés à la migration (Rossier, Ducarroz, 2012).

Nos résultats indiquent par ailleurs que les enfants des zones non loties souffrent d'un désavantage important dans le domaine de la santé : l'en- 
vironnement y est nettement plus insalubre, les enfants y ont plus de fièvres, et y sont plus souvent malnutris. Parce que les migrants habitent plus souvent en non loti, ils exposent leurs enfants aux risques inhérents à cet environnement. Les analyses bivariées ont d'ailleurs montré que les enfants de migrants ont un peu plus de fièvres et souffrent un peu plus de malnutrition, même si ces résultats ne sont pas significatifs. L'analyse future des différentiels de la mortalité des enfants, qui sera possible quand l'OPO aura collecté plus de cas de décès, permettra d'examiner si les enfants de migrants présentent un désavantage du point de vue de la mortalité, et si cet éventuel désavantage s'explique par leur insertion résidentielle en milieu non loti. Le cas de Ouagadougou serait alors semblable aux résultats de Brockerhoff (1990), qui a montré à partir des données d'EDS au Sénégal, que l'excédent de mortalité des enfants de migrantes est concentré dans les quartiers insalubres des villes.

En ce qui concerne les adultes, les migrants sont moins déprimés par rapport aux natifs de la capitale, toutes choses égales par ailleurs. Ces résultats confirment ceux d'une étude longitudinale récente en Thaïlande où les migrants du milieu rural jouissent d'une meilleure santé mentale, comparés aux natifs de la ville (Nauman et al., 2011). Ces auteurs expliquent ce résultat par le fait que les migrants gardent certains aspects de leur mode de vie rural qui les rendent plus résistants aux stress de la ville (un phénomène de «non acculturation»). D'autres résultats de l'OPO témoignent d'effets de «non acculturation» : la moindre utilisation contraceptive des migrants (Rossier, Ortiz, 2011), et le moindre recours aux soins des adultes migrants quelle que soit leur durée de résidence en ville (Nikiema et al., 2011). Une autre hypothèse discutée par Nauman et al. (2011) est celle du retour au village des migrants en moins bonne santé mentale. Nos résultats pourraient aller dans le sens de cette dernière hypothèse : seuls les migrants plus anciens semblent en effet jouir d'un avantage en termes de santé mentale. Alternativement, la propension moindre des résidents de longue durée à déprimer pourrait s'expliquer par le fait que les effets perturbateurs de l'installation font perdre momentanément aux migrants récents leurs avantages par rapport à la santé mentale.

Les études menées dans d'autres villes africaines ont montré que les migrants étaient protégés de l'obésité par de bonnes habitudes alimentaires à leur arrivée en ville, avantage qui se dissiperait avec le temps (Sodjinou, 2008 ; Duboz et al., 2011 pour une revue complète de cette littérature). On ne constate pas un tel phénomène dans les quartiers de l'OPO : les migrants par rapport aux natifs ne sont pas protégés du surpoids, 
toutes choses égales par ailleurs, et quelle que soit leur durée de résidence. Il est possible que notre variable de durée ne soit pas assez fine pour capturer un éventuel avantage des migrants juste après leur arrivée. Il est possible également que l'intégration sociale rapide des migrants constatées dans les quartiers de l'OPO se traduise par une adoption rapide de nouveaux comportements alimentaires dès que le statut socio-économique du migrant le lui permet.

Notre analyse montre que les adultes migrants ont un risque d'hypertension plus élevé que les natifs de Ouagadougou, mais seulement après avoir résidé longuement en ville. Ce résultat est contraire aux attentes de la littérature : les études existantes en Afrique montrent que les migrants souffrent moins souvent d'hypertension à leur arrivée en ville, mais ont avec le temps un risque d'hypertension comparable aux natifs (voir Duboz et al., 2011 pour une revue complète de cette littérature). On peut écarter dans notre cas l'hypothèse d'une migration liée à l'hypertension, c'est-à-dire le cas où des migrants souffrant de cette maladie viendraient se soigner en ville, puisque seuls les migrants plus anciens sont plus souvent hypertendus que les natifs. Par contre, il est possible que les personnes plus âgées ${ }^{9}$ et originaires du milieu rural retournent plus souvent au village au moment de leur retraite s'ils n'ont pas de problèmes cardiovasculaires ${ }^{10}$. Un tel effet de sélection pourrait expliquer ce résultat non attendu.

Enfin, nous observons que les migrants ont moins d'accidents que les natifs, ce résultat étant significatif seulement pour les résidents de longue durée. Comme déjà mentionné, d'autres analyses de l'enquête santé ont montré que les migrants recourent moins souvent aux soins en cas de maladie, quelle que soit leur durée de résidence (Nikiema et al., 2011). Il est donc probable que cette moindre exposition des migrants aux accidents soit un artefact de déclaration, puisqu'il s'agissait ici des accidents ayant donné lieu à un recours aux soins. L'absence de relation significative chez les migrants de courte durée pourrait s'expliquer par le fait que l'effet de leur moindre recours aux soins est contrebalancé par une fréquence plus élevée d'accidents, ce qui irait dans le sens de l'hypothèse des effets perturbateurs liés à la migration.

9. Les personnes de plus de 50 ans constituent $38 \%$ de l'échantillon des adultes enquêtés à l'enquête santé.

10. On observe souvent à Ouagadougou un retour des migrants au village après la retraite. On fait l'hypothèse que ceux ayant des maladies cardiovasculaires retourneraient moins, étant donné que la prise en charge de ces maladies est plus aisée en ville. 


\section{Conclusion}

Malgré ses contraintes et ses limites, cette étude montre que les enfants de migrants à Ouagadougou sont en aussi bonne santé (risques de malnutrition et de fièvre) que les enfants de natifs, quelle que soit la durée de résidence de leur mère. De plus, les pratiques d'assainissement (gestion des ordures et type de toilette), facteurs de maladies infectieuses chez les enfants, semblent tout aussi bonnes dans les ménages dirigés par des migrants. Chez les adultes par contre, la santé des migrants diffère de celle des natifs. Les migrants ayant résidé plus de 10 ans en ville sont en effet avantagés par rapport aux natifs en ce qui concerne la dépression. À l'inverse, contrairement aux études menées dans d'autres villes africaines, les migrants de Ouagadougou ne sont pas moins souvent en surpoids ou moins souvent hypertendus à leur arrivée. Dans le cas de l'hypertension, ils sont même plus à risque que les natifs après 10 années de résidence. Ces migrants de longue durée sont moins à risque d'accidents ayant donné lieu à un recours aux soins, un résultat qui peut être attribué à leur moindre propension à consulter en cas de problème de santé.

Le fait que la santé des migrants ne soit pas moins bonne que celle des natifs de Ouagadougou semble témoigner de la rapide intégration sociale des migrants dans cette ville. Toutefois, on ne peut pas exclure que ce résultat soit lié au manque de finesse de notre mesure de la durée en ville ou au retour au village des migrants qui s'adaptent moins vite. La dépression est le seul domaine où les migrants semblent se distinguer fondamentalement des urbains, par un avantage qui ne disparait pas avec le temps. Les phénomènes de «non acculturation» des migrants gagneraient peut-être à être étudiés pour certaines conditions de santé. Mais le résultat avec la dépression suggère également un effet de sélection probable des migrants : les migrants les plus déprimés retournent peut-être plus souvent au village, laissant les moins déprimés en ville. De manière encore plus frappante, la surreprésentation des hypertendus parmi les migrants résidant depuis longtemps en ville pourrait être liée à un moindre retour au village des migrants malades après leur retraite

Pour explorer plus avant ces différentes relations, il est nécessaire de dépasser l'approche transversale et de collecter des données longitudinales qui tiennent compte des événements de santé avant l'arrivée des migrants en ville et, de manière plus originale, de ce qui se passe après leur retour pour ceux qui n'y restent pas. Une étude à Ouagadougou a montré qu'une part importante des migrants quitte la ville, plus ou 
moins rapidement : après 10 ans, il ne reste plus que la moitié des migrants (Schoumaker et al., 2002). L'étude de l'effet du statut migratoire de la mère sur la mortalité des enfants dans les bidonvilles de Nairobi (Bocquier et al., 2011) utilisant des données longitudinales, tient compte des migrants qui repartent. Ses résultats montrent qu'une analyse transversale sous-estime le risque de mortalité des enfants de migrants, puisque ce sont les familles en plus mauvaise santé qui repartent le plus vite.

\section{Références}

Акото Е. (1990), «Christianisme et inégalités en matière de mortalité des enfants en Afrique noire», Population, 45 (6), pp. 971-992.

Beauchemin C., SChoumaker B. (2005), «Migration to Cities in Burkina Faso: Does the Level of Development in Sending Areas Matter?», World Development, 33 (7), pp. 1129-1152.

Bocquier P., Beguy D., Zulu E., Muindi K., Konseiga A., Yé Y. (2011), «Do Migrant Children Face Greater Health Hazards in Slum Settlements? Evidence from Nairobi, Kenya», Journal of Urban Health, 88, pp. 266-281.

Boyer F., Delaunay D. (2009), Peuplement de Ouagadougou et développement urbain, Rapport de recherche, IRD, Ouagadougou, $250 \mathrm{p}$.

BRIEND A. (1998), La malnutrition de l'enfant: des bases physiopathologiques à la prise en charge sur le terrain, Institut Danone, Bruxelles, $163 \mathrm{p}$.

BROCKERHOFF M. (1990). «Rural-to-Urban Migration and Child Survival in Senegal», Demography, 27 (4), pp. 601-615.

BrocKerHOFF M. (1994), «The Impact of Rural-Urban Migration on Child Survival», Health Transition Review, 4 (2), pp. 127-149.

Dibley M. J., Staehling N., Nieburg P., Trowbridge F. L. (1987), «Interpretation of ZScore Anthropometric Indicators Derived from the International Growth Reference», American Journal of Clinical Nutrition, 46, pp. 749-762.

Duboz P., Macia E., Gueye L., Chapuis-Lucciani N. (2011), «Migrations internes et santé à Dakar (Sénégal). Comparaison de l'autoévaluation de la santé, de l'hypertension artérielle et de l'obésité des migrants internes et des personnes originaires de Dakar», Bulletins et Mémoires de la Société d'Anthropologie de Paris, 23 (1-2), pp. 83-93.

DUthÉ G., Bonnet D., Rossier C. (2013), «La santé mentale, un problème négligé de la transition sanitaire : le cas de la dépression dans les quartiers périphériques de Ouagadougou», Working Paper du CEPED, Numéro 29, UMR 196 CEPED, Université Paris Descartes, INED, IRD, Paris, www.ceped.org/wp. 
Filmer D., Pritchett L. (1999), «The Effect of Household Wealth on Educational Attainment: Evidence from 35 Countries», Population and Development Review, 25 (1), pp. 155-174.

Filmer D., Pritchett L. (2001), «Estimating Wealth Effects without Expenditure Data or Tears: An Application to Educational Enrolments in States of India», Demography, 38 (1), pp. 115-132.

Guengant J. P. (2009), "Évolution passée et future de la ville de Ouagadougou», Peuplement de Ouagadougou et développement urbain, F. BOYE, D. Delaunay (eds), Rapport provisoire, IRD, Ouagadougou, pp. 42-49.

LACHAUd J.P. (2001), Dynamique de pauvreté et inégalité de la mortalité des enfants au Burkina Faso, Centre d'Économie du Développement de l'Université Montesquieu-Bordeaux IV, Bordeaux, $21 \mathrm{p}$.

KIRos G. E., White M. J. (2004), «Migration, Community, Context and Child Immunization in Ethiopia», Social Sciences and Medicine, 59 (12), pp. 2603-2616.

Konseiga A., Zulu E., Bocquier P., Muindı K., Beguy D., Yé Y. (2009), «Assessing the Effect of Mother's Migration on Chilhood Mortality in the Informal Settlement of Nairobi", The Dynamics of Migrations, Health and Livelihoods, M. ColuINSON, K. AdAZU, M. White, S. Findley (eds), ASHGATE, Farnham \& Burlington, pp. 123-138.

MARIKo S. (2004), "Allaitement et état nutritionnel», Enquête Démographique et de Santé 2003, Burkina Faso, Institut National de la Statistique et de la Démographie, INSD et Macro International, Ouagadougou et Claverton, pp. 145-172.

Miranda J. J., Gilman R. H., Garcia H. H., Smeeth L. (2009), «The Effect on Cardiovascular Risk Factors of Migration from Rural to Urban Areas in Peru: PERU MIGRANT Study", BMC Cardiovascular Disorders, 9 (23), $16 \mathrm{p}$.

Montgomery M. R., Gragnolatı M., Burke K. A., Paredes E. (2000), «Measuring Living Standards with Proxy Variables», Demography, 37 (2), pp. 155-174.

Nauman E., Van landingham M., Anglewicz P., Patthavanit U., Punpuing S. (2011), Rural-to-Urban Migration and Changes in Health Among Young Adults in Thailand, Communication à la PAA 2011, Washington, $20 \mathrm{p}$.

Nikiema A., Millogo R., Rossier C., Ridde V. (2011), Inégalités de l'accès aux soins en milieu urbain africain : le cas de la périphérie Nord de Ouagadougou, Communication présentée à la 6ème Conférence de la Population Africaine, Ouagadougou, 59/12/11, http://uaps2011. princeton.edu/sessionViewer.aspx?Sessionld=805.

Organisation Mondiale de LA SANTE (2008), 61e Assemblée mondiale de la Santé : de nouveaux objectifs ambitieux pour l'OMS, Communiqué de Presse, www.who.int/ mediacentre/news/releases/2008/wha02/fr/ (site visité le 8/01/2013).

Rossier C., Soura A., Lankoande B., Millogo R. (2011), Observatoire de Population de Ouagadougou. Données collectées au Round 0, Round 1 et au Round 2, Rapport descriptif, ISSP, Ouagadougou, $71 \mathrm{p}$. 
Rossier C., Soura A., Baya B., Compaore G., Dabire B., Dos Santos S., Duthe G., Gnoumou B., Kobiane J.-F., Kouanda S., lankoande B., Legrand T., Mbacke C., Millogo R., Mondain N., Montgomery M., Nikiema A., Ouil I., Pison G., Randall S., SANGl G., Schoumaker B., Zourkhaleini Y. (2012), "The Ouagadougou Health and Demographic Surveillance System», International Journal of Epidemiology, 41 (3), pp. 658-666.

Rossier C., DuCARroz L. (2012), La pauvreté dans les quartiers de l'OPO : une approche qualitative, Rapport de recherche, ISSP, Ouagadougou, $76 \mathrm{p}$.

Rossier C., Ortiz I. (2011), Unmet Needs for Contraception in Formal and Informal Neighborhoods of Ouagadougou, Communication à la 6ème Conférence africaine sur la population, 5-9/12/11.

Sheehan D.-C., LeCRubier Y. (1998), «The Mini International Neuropsychiatric Interview (MINI): The Development and Validation of a Structured Diagnostic Psychiatric Interview for DSMIV and ICD-10», J. Clin. Psychiatry, 59 (20), pp. 22-33.

Schoumaker B., Beauchemin C., Dabire B., (2002), "Les migrations à Ouagadougou : tendances récentes (1990-2000)», Ouaga Focus $n^{\circ} 30$, UERD, Ouagadougou, 2 p.

Sodjinou R., Agueh V., Fayomı B., Delisle H. (2008), "Obesity and Cardio-Metabolic Risk Factors in Urban Adults of Benin: Relationship with Socio-Economic Status, Urbanisation, and Lifestyle Patterns», BMC Public Health, 8 (84), 13 p.

Stephenson R., Matthews Z., McDonald J. W. (2003), "The Impact of Rural-Urban Migration on Under-two Mortality in India», Journal of Biosocial Science, 35 (1), pp. 15-31.

Ssengonzi R., De Jong G. F., Stokes C. S. (2002), «The Effect of Female Migration on Infant and Child Survival in Uganda», Population Research and Policy Review, 21, pp. 403-431.

SubaiYa L. (2007), Internal Migration and the Use of Reproductive and Child Health Services in Peru, DHS Working paper, 38, Macro International Inc, Calverton, $17 \mathrm{p}$.

Zourkaleinı Y., PICHÉ V. (2007), «Economic Integration in West-African Urban Labour Market: Does Migration Matter? The Case of Ouagadougou, Burkina Faso», Demographic Research, 17, pp. 497-450. 